\title{
Efficacy of Dupilumab for Atopic Dermatitis According to Clinical Course and Clinical Findings: A Multicentre Retrospective Study
}

Takeshi NAKAHARA ${ }^{1}$, Makiko KIDO-NAKAHARA ${ }^{1}$, Daisuke ONOZUKA ${ }^{2}$, Sawako SAKAI ${ }^{1}$, Tomoko HIROSE ${ }^{3}$, Nobutoshi TAKE ${ }^{3}$, Akiko SUGIYAMA ${ }^{4}$, Kayo HARADA ${ }^{5}$, Gaku TSUJI ${ }^{1}$, Satoko KIKUCHI ${ }^{6}$ and Futoshi KOHDA ${ }^{7}$

${ }^{1}$ Department of Dermatology, Graduate School of Medical Sciences, Kyushu University, 3-1-1 Maidashi, Higashi-ku, Fukuoka 812-8582, ${ }^{2}$ Department of Medical Informatics and Clinical Epidemiology, Graduate School of Medical Science, Kyoto Prefectural University of Medicine, Kyoto, Departments of Dermatology, ${ }^{3}$ Kitakyushu Medical Center, Kitakyushu, ${ }^{4}$ Fukuoka National Hospital, ${ }^{5} \mathrm{Kyushu}$ Medical Center, ${ }^{6} \mathrm{Kyushu}$ Central Hospital, Fukuoka and 'Iizuka Hospital, Iizuka, Japan. E-mail: nakahara@dermatol.med.kyushu-u.ac.jp

Accepted Oct 28, 2021; Epub ahead of print Oct 28, 2021

Adult patients with atopic dermatitis (AD) can be divided into 3 groups, based on onset and clinical course: $(i)$ continuous type, with onset in childhood and relapses and improvements of the condition throughout life; (ii) recurrent type, with a typical childhood history of AD that is outgrown but recurs later in life; and (iii) adultonset type $(1,2)$. AD is mainly suspected in flexural and symmetrical eczema, and this clinical presentation is often similar in children and adults. However, some forms of $\mathrm{AD}$ are more specific to adults, including a type with severe symptoms on the face, neck, upper chest, and upper back area (facial type AD), and a type with prurigo (prurigo type AD) (1-3). Although AD is a heterogeneous disease, few studies have investigated the effects of dupilumab according to the clinical course or clinical findings. Thus, the aim of the current study was to investigate the clinical outcomes of dupilumab treatment for $\mathrm{AD}$ in patients with different clinical courses or clinical findings.

\section{MATERIALS AND METHODS}

This was a multicentre retrospective survey of 116 adult patients with $\mathrm{AD}$ (age $>15$ years) treated with dupilumab. The 116 patients with AD were divided into 3 groups based on clinical course (continuous type, $n=75$; recurrent type, $n=23$; adult-onset type, $n=18$ ) or clinical findings (general type, $n=33$; facial type, $n=69$; prurigo type, $n=14$ ). It was difficult to classify clinical findings in detail; hence facial type and prurigo type $\mathrm{AD}$ were distinguished as special types, and all other clinical findings were grouped together as "general" type. Patient background information and AD-related clinical data for each group are provided in Table SI". The Eczema Area and Severity Index (EASI; total EASI score and the head and neck (HN)-EASI subscore), Patient-Oriented Eczema Measure (POEM), and Itch Numerical Rating Scale (I-NRS) were examined before and 16 weeks after the start of dupilumab treatment. A mixed-effects linear regression model was used to evaluate associations of treatment duration with total EASI, HNEASI, POEM, and I-NRS scores, adjusted for clinical course or clinical findings. Coefficients and 95\% confidence intervals $(95 \%$ CIs) were calculated; in these analyses, a coefficient of 0 indicates the same improvement as seen in the reference group, whereas positive and negative values indicate less and more improvement, respectively. Statistical significance was set at 2 -sided $p<0.05$.

${ }^{1}$ https://doi.org/10.2340/actadv.v101.369

\section{RESULTS}

For the entire patient cohort, there were significant improvements in the total EASI, HN-EASI, POEM, and I-NRS scores after 16 weeks of treatment with dupilumab (Fig. S1, Table SII ${ }^{1}$ ). Similarly, significant improvements were seen in all scores for each of the clinical course (Fig. S2A, Table SIII ${ }^{1}$ ) and clinical findings (Fig. S2B, Table SIV ${ }^{1}$ ) groups when analysed separately. Because the clinical course and clinical findings may affect the efficacy of dupilumab, we adjusted for these potential effects to examine the efficacy of dupilumab in mixedeffects linear regression analyses. Even after adjusting for clinical course (Table I) or clinical findings (Table II), dupilumab treatment for 16 weeks significantly improved the scores of all AD-related parameters. The current study also investigated whether there were differences in the improvement in each score between groups (Tables I and II). In comparisons of the clinical course groups, there were no significant differences for any of the scores among groups (Table I). However, comparisons of changes in total EASI, HN-EASI, POEM, and I-NRS

\begin{tabular}{|c|c|c|c|}
\hline Characteristics & Coefficient & $95 \% \mathrm{CI}$ & $p$-value \\
\hline \multicolumn{4}{|c|}{ Total Eczema Area and Severity Index } \\
\hline Week & -1.25 & $-1.39,-1.10$ & $<0.001$ \\
\hline \multicolumn{4}{|l|}{ Clinical course } \\
\hline Continuous type & Reference & & \\
\hline Recurrent type & -2.36 & $-7.37,2.65$ & 0.355 \\
\hline Adult-onset type & -3.96 & $-9.38,1.46$ & 0.152 \\
\hline \multicolumn{4}{|c|}{ Head and Neck Eczema Area and Severity Index } \\
\hline Week & -0.12 & $-0.14,-0.11$ & $<0.001$ \\
\hline \multicolumn{4}{|l|}{ Clinical course } \\
\hline Continuous type & Reference & & \\
\hline Recurrent type & -0.27 & $-0.86,0.33$ & 0.374 \\
\hline Adult-onset type & -0.56 & $-1.23,0.11$ & 0.099 \\
\hline \multicolumn{4}{|c|}{ Patient-Oriented Eczema Measure } \\
\hline Week & -0.74 & $-0.83,-0.66$ & $<0.001$ \\
\hline \multicolumn{4}{|l|}{ Clinical course } \\
\hline Continuous type & Reference & & \\
\hline Recurrent type & -0.60 & $-2.84,1.63$ & 0.596 \\
\hline Adult-onset type & 1.44 & $-0.99,3.88$ & 0.246 \\
\hline \multicolumn{4}{|c|}{ Itch Numerical Rating Scale } \\
\hline Week & -0.26 & $-0.30,-0.22$ & $<0.001$ \\
\hline \multicolumn{4}{|l|}{ Clinical course } \\
\hline Continuous type & Reference & & \\
\hline Recurrent type & -0.10 & $-0.90,0.71$ & 0.817 \\
\hline Adult-onset type & 0.12 & $-0.85,1.08$ & 0.812 \\
\hline
\end{tabular}


Table II. Changes in atopic dermatitis-associated parameters after 16 weeks dupilumab treatment following adjustment for clinical findings

\begin{tabular}{|c|c|c|c|}
\hline Characteristics & Coefficient & $95 \% \mathrm{CI}$ & $p$-value \\
\hline \multicolumn{4}{|c|}{ Total Eczema Area and Severity Index } \\
\hline Week & -1.25 & $-1.39,-1.10$ & $<0.001$ \\
\hline \multicolumn{4}{|l|}{ Clinical findings } \\
\hline General type & Reference & & \\
\hline Facial type & 5.34 & $1.02,9.66$ & 0.015 \\
\hline Prurigo type & 5.04 & $-1.56,11.63$ & 0.134 \\
\hline \multicolumn{4}{|c|}{ Head and Neck Eczema Area and Severity Index } \\
\hline Week & -0.12 & $-0.14,-0.11$ & $<0.001$ \\
\hline \multicolumn{4}{|l|}{ Clinical findings } \\
\hline General type & Reference & & \\
\hline Facial type & 0.62 & $0.12,1.11$ & 0.015 \\
\hline Prurigo type & -0.73 & $-1.51,0.05$ & 0.066 \\
\hline \multicolumn{4}{|c|}{ Patient-Oriented Eczema Measure } \\
\hline Week & -0.74 & $-0.82,-0.65$ & $<0.001$ \\
\hline \multicolumn{4}{|l|}{ Clinical findings } \\
\hline General type & Reference & & \\
\hline Facial type & 1.09 & $-0.90,3.07$ & 0.283 \\
\hline Prurigo type & 0.01 & $-2.99,3.00$ & 0.996 \\
\hline \multicolumn{4}{|c|}{ Itch Numerical Rating Scale } \\
\hline Week & -0.26 & $-0.30,-0.22$ & $<0.001$ \\
\hline \multicolumn{4}{|l|}{ Clinical findings } \\
\hline General type & Reference & & \\
\hline Facial type & -0.32 & $-1.06,0.42$ & 0.401 \\
\hline Prurigo type & 0.06 & $-1.04,1.16$ & 0.915 \\
\hline
\end{tabular}

95\% CI: 95\% confidence interval.

scores according to clinical findings (Table II), using the general type group as a reference group, revealed significantly greater improvements in the general type than facial type group for both total EASI score (coefficient 5.34; 95\% CI 1.02, 9.66; $p=0.015)$ and HN-EASI score (coefficient $0.62 ; 95 \%$ CI $0.12,1.11 ; p=0.015$ ). There were no significant differences in any other comparisons among groups (Table II).

\section{DISCUSSION}

In this study, dupilumab significantly improved scores for all AD-related measures in all groups classified by clinical course and clinical findings. This suggests that interleukin (IL)-4 and IL-13 are central to the pathogenesis of all forms of AD. In particular, there were no significant differences in improvements in each of the scores among groups classified based on clinical course. Age-related differences in T cell subsets involved in AD pathogenesis have been reported (4). However, there may be no significant difference in the involvement of IL-4 and IL-13 in the pathogenesis of adult AD, even though the clinical course may differ. In comparisons of improvements in the scores between clinical findings groups, improvements in the total EASI and HN-EASI scores were significantly greater in the general type than in the facial type group. Both the face and neck are exposed to the outside world, and factors other than IL-4 and IL-13, such as contact irritation and infection, are more likely to be involved in skin rashes (5). The involvement of factors other than IL-4 and IL-13 in its pathogenesis may be a feature of facial type $\mathrm{AD}$, and may explain the slower improvement in scores following dupilumab treatment of patients with facial AD. Nevertheless, there were significant improvements in both total EASI and HN-EASI scores even among patients with facial type $\mathrm{AD}$; hence, an even greater improvement may be seen when dupilumab is combined with topical agents that have different mechanisms of action other than IL-4 and IL-13. Considering that prurigo has been difficult to treat with existing therapies, the high efficacy of dupilumab in the treatment of prurigo demonstrates its usefulness. POEM and I-NRS, which are assessed from a patient's perspective $(6,7)$, improved similarly in all clinical finding groups. These results suggest that dupilumab is a suitable treatment from the patient's perspective in terms of continuation of treatment, long-term control, and achieving treatment goals.

In conclusion, this study shows that dupilumab is likely to be an effective treatment for $\mathrm{AD}$, regardless of the clinical course and clinical findings, although improvements in total EASI and HN-EASI scores are somewhat slower for patients with facial AD.

\section{ACKNOWLEDGEMENTS}

The authors thank all the members who collected clinical data. Conflicts of interest: TN received research funding from Maruho and Sanofi Corporation. The remaining authors have no conflicts of interest to declare.

\section{REFERENCES}

1. Chello C, Carnicelli G, Sernicola A, Gagliostro N, Paolino G, Di Fraia $M$, et al. Atopic dermatitis in the elderly Caucasian population: diagnostic clinical criteria and review of the literature. Int J Dermatol 2020; 59: 716-721.

2. Silvestre Salvador F, Romero-Pérez D, Encabo-Durán B. Atopic dermatitis in adults: a diagnostic challenge. J Investig Allergol Clin Immunol 2017; 27: 78-88.

3. Hello M, Aubert H, Bernier C, Néel A, Barbarot S. Atopic dermatitis of the adult. Rev Med Interne 2016; 37: 91-99.

4. Czarnowicki T, He H, Krueger JG, Guttman-Yassky E. Atopic dermatitis endotypes an implications for targeted therapeutics. J Allergy Clin Immunol 2019; 143: 1-11.

5. Tamagawa-Mineoka R, Katoh N. Atopic dermatitis: identification and management of complicating factors. Int $\mathrm{J} \mathrm{Mol} \mathrm{Sci}$ 2020; $21: 2671$.

6. Thomas KS, Apfelbacher CA, Chalmers JR, Simpson E, Spuls PI, Gerbens LAA, et al. Recommended core outcome instruments for health-related quality of life, long-term control and itch intensity in atopic eczema trials: results of the HOME VII consensus meeting. Br J Dermatol 2021; 185: 139-146.

7. Chalmers JR, Simpson E, Apfelbacher CJ, Thomas KS, von Kobyletzki L, Schmitt J, et al. Report from the fourth international consensus meeting to harmonize core outcome measures for atopic eczema/dermatitis clinical trials (HOME initiative). Br J Dermatol 2016; 175: 69-79. 